\title{
¡EUREKA! SE ACELERAN LOS CAMBIOS ORGANIZACIONALES CON LA PANDEMIA
}

\author{
Kobila, María Teresa \\ Parolin, Marisa Andrea \\ D'Avanzo, María Laura \\ Chiaramoni, Natalia Soledad \\ Gagliardini, Graciela Ana
}

\section{Resumen:}

En la vertiginosidad actual la sociedad y las organizaciones están viviendo momentos de incertidumbre, riesgo y caos. Nos preguntamos ¿cómo se encuentran las organizaciones de cara a la pandemia? ¿Cómo reaccionan y deciden los directivos? ¿Qué rol ocupan los líderes? ¿Qué actitudes asumen? ¿Cómo responden los colaboradores? ¿Se implementan nuevas estrategias y prácticas de trabajo? ¿Cómo se asumen los nuevos cambios? Proponemos indagar los modos de entender y asumir el cambio por parte de los directivos y/o líderes empresariales, narrar las modalidades de trabajo y formas de adaptación de sus miembros en respuesta a los nuevos escenarios y comparar los tipos de empresas procurando realizar una primera aproximación del cambio organizacional en el presente. Resultó que el mayor escollo derivó del impedimento para trabajar, no por falta de adaptabilidad de las personas a la tecnología.

La supervivencia de las organizaciones depende de varios factores, no solo de la capacidad de gestión en situaciones de crisis, por lo tanto no es factible aseverar la carencia de capacidades de conducción de sus directivos y/o líderes. En el mundo de las empresas y el trabajo, los cambios se aceleraron por y con la pandemia, dejando numerosos interrogantes en torno al impacto Pospandemia. ¿Podrán las organizaciones en crisis recuperarse? Las que queden en pie ¿cómo se verán afectadas en sus estrategias, culturas y estructuras?

Palabras clave: Pandemia - Cambio organizacional - Estilos directivos - Nueva realidad.

\section{ABSTRACT:}

In the vertiginous present, society and organizations are living moments of uncertainty, risk and chaos. We question how organizations are in the face of the pandemic? How do managers react and decide? What role do leaders play? What attitudes do they assume? How do collaborators respond? Are new work strategies and practices implemented? How are the new changes assumed? We propose to investigate the ways of understanding and assuming the change on the part of the managers and / or 
business leaders, narrate the working modalities and forms of adaptation of its members in response to the new scenarios and compare the types of companies trying to make a first approximation of organizational change in the present. It turned out that the greatest difficulty derived from the impediment to work, not from lack of adaptability of people to technology.

The survival of organizations depends on several factors, not only on the capacity to manage in crisis situations; therefore it is not feasible to assert the lack of leadership skills of their managers and / or leaders. In the world of business and work, changes were accelerated by and with the pandemic, leaving many questions about the Postpandemic impact. Can organizations in crisis recover? Those that remain, how will their strategies, cultures and structures be affected?

Key words: Pandemic - Organizational changes - Manager's styles - New reality.

\section{INTRODUCCIÓN}

En las últimas décadas el ritmo del cambio se ha acelerado. En la vertiginosidad actual la sociedad y las organizaciones están viviendo momentos de incertidumbre, riesgo y caos. El aceleramiento de las transformaciones obliga a las organizaciones a adoptar nuevas estrategias, políticas y prácticas para sobrevivir en un mercado tan competitivo como complicado.

En medio de este panorama, como para echar leña al fuego, surge un fenómeno nuevo e inesperado: la pandemia por el Covid-19 que impacta en lo sanitario, social, político y económico y en principio, paralizó al mundo.

El costo económico y social de los esfuerzos que deben desplegarse para detener la pandemia es innegable. En estas circunstancias, la OIT ha defendido la adopción de respuestas a la COVID-19 basadas en cuatro pilares: estimular la economía y el empleo; apoyar a las empresas, los empleos y los ingresos; proteger a los trabajadores en el lugar de trabajo y recurrir al dialogo social para encontrar soluciones (OIT, julio 2020).

En este medio, las organizaciones y particularmente el mundo del trabajo se vieron gravemente afectados con secuelas para empresarios, directivos y trabajadores. Nuestro trabajo como docentes e investigadoras del campo de la Administración no puede permanecer ajeno a esta realidad.

El nuevo virus golpea al mundo y ha puesto en discusión la necesidad de nuevos estilos mundiales de liderazgos, liderazgos más colaborativos. "Es obvio que nos falta el liderazgo que solo puede ser posible si (...) las potencias mundiales claves son capaces de aproximarse, adoptar una estrategia común y luego reunir a toda la 
comunidad internacional" (BBC, 05 de mayo 2020), admitió el secretario general de la ONU, António Guterres.

Debate que también ha comenzado a instalarse a nivel microeconómico. Varios directivos y empresarios perciben estas circunstancias como verdaderos desafíos adoptando medidas tendientes a cuidar a sus trabajadores, conservar y atraer clientes y mantener buenas relaciones con sus proveedores y otros grupos de interés. Son tiempos de transformación que requiere de líderes proactivos y de colaboradores comprometidos. La clave radica en gestionar adecuadamente el cambio.

Frente a esta realidad nos preguntamos ¿cómo se encuentran las organizaciones de cara a la pandemia? ¿Cómo reaccionan y deciden los directivos? ¿Qué rol ocupan los líderes? ¿Qué actitudes asumen? ¿Cómo responden los colaboradores? ¿Se implementan nuevas estrategias y prácticas de trabajo? ¿Cómo se asumen los nuevos cambios?

Estas primeras inquietudes nos llevaron a bucear en el nuevo escenario organizacional para indagar ¿qué está pasando en el mundo de las empresas y el trabajo?

Las palabras clave que convocan son: Pandemia - Cambio organizacional - Estilos directivos - Nueva realidad.

\section{OBJETIVOS}

- Indagar los modos de entender y asumir el cambio por parte de los directivos y/o líderes empresariales. .

- Narrar las modalidades de trabajo y formas de adaptación de sus miembros en respuesta a los nuevos escenarios.

- Comparar los tipos de empresas procurando realizar una primera aproximación del cambio organizacional en el presente.

\section{METODOLOGÍA}

El estudio, de carácter descriptivo, tiene como punto de partida la lectura de opiniones de empresarios rosarinos en escenario presente. El relevamiento de datos se efectuó mediante entrevistas a través de medios virtuales (meet y zoom) mediante encuentros que fueron acordados previamente por email o a través de whatsapp. La recopilación de información se efectuó entre el 15 de mayo y el 15 de julio del año 2020. En total se efectuaron diecisiete (17) entrevistas a responsables de diferentes tipos de organizaciones de la ciudad de Rosario.

Se empleó la técnica de muestreo de tipo no probabilístico -por conveniencia- para la selección de los casos ya que los mismos se lograron gracias a contactos personales 
del equipo de investigadoras y atento a las posibilidades reales de concesión. Las entrevistas por videoconferencia, como prueba piloto, resultaron experiencias de aprendizaje y primeros contactos entre entrevistador/a y equipo de investigación.

Como ventajas de esta modalidad se destaca la flexibilidad horaria para los encuentros y economía de costos dado que no hubo que trasladarse hasta los lugares en que se emplazan los casos de estudio. Como debilidad se detecta que se pierde la riqueza del encuentro presencial, es decir que se pierde la observación personal considerada como una herramienta valiosa para los estudios cualitativos.

Es importante recalcar que, a pedido de los entrevistados, se preservó la identidad de los informantes y sus empresas. También, se está solicitando autorización para continuar con el relevamiento de los empleados en estas mismas organizaciones, a las cuales agradecemos la información suministrada.

La exploración permitió obtener una visión amplia sobre el modo que los entrevistados conciben y reaccionan frente a la situación de cambio. Esta primera aproximación al terreno dejó las puertas abiertas para profundizar la indagación y obtener información más detallada a posteriori.

Además, resultó valioso el trabajo realizado porque posibilitó orientar el rumbo de nuestra investigación, dado que el tema se enmarca en un proyecto de mayor envergadura sobre "Gestión de la Complejidad Cultural en las Organizaciones" radicado en la Facultad de Ciencias Económicas y Estadística de la Universidad Nacional de Rosario (U.N.R.).

\section{REFERENCIAL TEORICO}

Es frecuente enunciar que el cambio organización refiere a "cualquier modificación de su personal, estructura o tecnología" (Robbins y Coulter; 2019:208) que puede producirse en forma planeada o emergente. La definición es clara y categórica, pero cuando se penetra en las formas del cambio y los impactos que pueden generar en las organizaciones, se torna en un tema de debate. El cambio desafía a empresarios, directivos y/o líderes que tienen la responsabilidad y necesidad de llevar a cabo transformaciones en la organización (Chiavenato, 2000).

Los cambios organizacionales son incuestionables, como también se requiere puntos de apoyo, bases que garanticen la estabilidad, aunque sea para ser removidos más tarde (Altschul y Carbonell, 2003), sin embargo, hoy, donde todo parece estar en estado de flujo, entender y explicar que es necesario cambiar genera ruidos.

Sostienen Hamel y Prahalad (1995) que cuando los cimientos de la industria están sufriendo sacudidas, las organizaciones necesitan tener una nueva perspectiva de lo que significa ser "estratégico" (Hamel y Prahald, 1995). Kotler (1996) cree que cuando 
el cambio es planificado se puede llevar a cabo por etapas y con un estilo de liderazgo transformador. Son indiscutibles estos aportes, y algunas organizaciones así lo transitan con resultados exitosos (Kobila y otros, 2019), pero cuando emerge algo impensado no hay recetas. Gestionar el cambio es imprescindible.

A partir del miércoles 11 de marzo de 2020 la Organización Mundial de la Salud (OMS) declara que el coronavirus COVID-19 como pandemia y en nuestro país, a partir del 20 de marzo el Gobierno mediante un decreto (DNU 297/20) dicta un aislamiento social preventivo y obligatorio. Desde entonces, todos los medios señalan que las personas experimentaron cambios en sus estructuras mentales y emocionales.

Imprevistamente, las organizaciones debieron afrontar el cambio instrumentando nuevas metodologías de trabajo remoto, nuevas tecnologías, nuevas demandas generadas, requiriendo de líderes competentes para la gestión. Hatum e Isola (agosto, 2020) describen este fenómeno organizacional apelando a la categoría de "reinvención de los recursos humanos", destacando el rol del área homónimo en la reorganización del trabajo y el cuidado de la salud física y emocional de sus colaboradores.

La pandemia ha obligado a las organizaciones a adaptarse a fuertes cambios incluso, en algunos casos, sin entender qué resguardar y qué cambiar. Algunas empresas hay tenido que invertir en tecnología, adoptando nuevas metodologías y procesos de trabajo para seguir siendo operativas; otras, tal vez se vieron tomando ciertas decisiones que en otro contexto, no se hubieran imaginado trabajar de esta manera. La virtualidad ha tenido sus adeptos, personas que se sintieron cómodas, otros se mostraron resistentes y también se hallan quiénes quedaron expuestos a sus debilidades.

Antes de la pandemia Molinari enunciaba "Liderar equipos en el cambio de y en una realidad líquida es una tarea compleja, que requiere entonces de un "self" con muchos recursos" (Molinari, 01 octubre, 2019), proponiendo: a) enfrentar los miedos con la cooperación, b) cuidarnos a nosotros, manteniendo el equilibrio y c) conectar con proyectos y con futuro, repensando el negocio (lbídem, 01/10/2019).

Hoy muchos directivos y/o líderes se cuestionan frente a qué realidad se enfrentan y si están preparados para gestionar en una incertidumbre de tal magnitud. ¿Qué líderes se necesitarán para las organizaciones en este nuevo contexto?

Molinari (2017) se anticipaba considerando que estamos en presencia de un cambio de paradigma, donde la representación del ámbito y la forma de trabajar se han alterado. Antes el espacio de trabajo era pensado como el "lugar al cual uno tenía que dirigirse", hoy, esa concepción se ha modificado, trabajar es lo que uno hace independientemente de "dónde lo hace". 
En esta nueva perspectiva la tecnología ganó protagonismo (Poe, 2009) y para muchos, el cambio es positivo porque permite equilibrar vida laboral y personal, lograr flexibilidad, libertad y mejor manejo del tiempo. Sin embargo, cambiar metodologías y procesos de trabajo implica un gran desafío para los directivos y/o líderes dado que implicaría pasar de un modelo de gestión por presencia a un modelo de gestión por resultados.

Según Molinari se debería pasar de un Modelo de Dirección y Control a un Modelo de Colaboración, procurando bienestar y cuidando la calidad de vida de los colaboradores. En este momento las organizaciones necesitan rediseñar sus espacios de trabajo, nuevos "lays outs" que parten de considerar al espacio como creación de valor (Ibídem, 2017).

Del mismo modo, Fabio Boggino (2015) juzga que estamos ante un nuevo paradigma donde el crecimiento de la tecnología en estos últimos años ha sido exponencial y se han desarrollado nuevas formas de comunicación a través de redes sociales, conexiones Wifi, celulares inteligentes, entre otros.

Boggino (2015), Molinari (2017), Melamed (2017) y otros coinciden en que el teletrabajo irrumpió en las organizaciones como una nueva modalidad de trabajo, requiriendo nuevas competencias en los perfiles de los líderes. No obstante el cambio, anticipado por los autores e impensado para muchos, precipitado con la pandemia requiere de la acomodación de las organizaciones y ello dependerá en gran medida de la capacidad de resiliencia y de los líderes que las conducen.

\section{RESULTADOS PRELIMINARES}

En este contexto de pandemia estamos en presencia de un cambio en el contexto y en las organizaciones. En este terreno, los resultados de esta ponencia ponen de manifiesto lo acaecido en algunas empresas locales, estimándose de gran valor como una primera aproximación al campo de estudio.

Como producto de la interpretación y análisis de la información recopilada, se elaboró una categorización de los casos estudiados.

\section{A) Aquellas que no han podido pudieron abrir sus puertas durante la pandemia y} aún no están autorizadas a desarrollar actividades. En este grupo se encuentran los hoteles, salones de fiesta, jardines de infantes. Si bien armaron protocolos de actuación, no han logrado aún su aprobación. Su panorama es incierto. Se endeudan día a día, tratando de cumplir con los compromisos asumidos, pagar sueldos y renegociar con sus empleados y acreedores la mejor manera de asignar los recursos. Algunas han intentado desarrollar actividades alternativas para subsistir. La titular de un jardín maternal expresó: 
"Nos podemos dar clases a los bebés vía zoom, algo tenemos que hacer para no cerrar. Las maestras se pusieron a fabricar juguetes que venden on line, los papás de los alumnos son nuestros principales clientes"

Se interpreta que en estas organizaciones, se requiere de un estilo de conducción resiliente capaz de trasmitir apoyo, optimismo y confianza en el equipo.

B) Los considerados rubros no esenciales y que pudieron abrir sólo temporalmente por autorización del gobierno nacional y/o local en función de las fases atravesadas. Entre ellas se cuentan tiendas, peluquerías, jugueterías, gimnasios, bares, compañías de seguro, estudios profesionales, entre otros. Ellas atravesaron la crisis con las puertas cerradas, sin público que pudiera acceder a comprar a sus locales y/o gozar de sus servicios en forma presencial. Debieron soportar todos los gastos fijos como sueldos, alquileres, impuestos. Una de las principales practicas implementadas, por algunas, fueron las ventas on-line, incursionando en el e-commerce. Así como los gimnasios implementaron clases virtuales. En este sentido, varios empresarios coincidieron en sus afirmaciones, a modo ejemplificativo se transcribe un comentario:

"Nunca habíamos vendido de manera virtual, no teníamos ni idea de cómo hacerlo...Nuestros hijos o los empleados más jóvenes nos ayudaron, incluso diseñaron páginas web y nosotros mismos repartimos los productos. La verdad es que descubrimos una nueva forma de llegar a los clientes, estamos acostumbrados a atenderlos personalmente. Por supuesto que sólo no alcanza pero es una ayuda...".

Es evidente que el cambio obligó a implementar nuevas prácticas antes impensadas. Para algunos, tal vez sea el puntapié inicial para entrar en nuevos nichos de mercados, aprovechando nuevas oportunidades. En tanto que otros se perciben interrumpidos, asumiendo un estilo de conducción pasiva, como dando tiempo al tiempo para ver qué pasa. También se notó con actitud pasiva y de resignación a muchos pequeños comerciantes, que asumen con desaliento el quiebre de sus empresas.

C) Empresas cuyas actividades son consideradas esenciales que permanecieron abiertas desde el primer día de la pandemia, entre ellas almacenes, supermercados, farmacias, ferreterías, empresas de vigilancia, de limpieza y las de servicios de emergencia y urgencias. El servicio de cadetería fue otro de los grandes demandados, por el incremento del servicio de delivery y el take-away.

\section{Para estos casos, la pandemia significó una oportunidad pero para aprovecharla}

tuvieron que tomar decisiones inmediatas. La mayoría obtuvieron más ventas, especialmente durante los primeros meses del confinamiento, y algunas debieron 
contratar personal para satisfacer la mayor demanda o bien, para cubrir puestos antes ocupados por personal considerado de riesgo como mayores de sesenta años, embarazas, etc. Asimismo reforzaron sus sistemas de ventas on line que aumentaron significativamente.

También debieron tomaron las medidas necesarias para cuidar la salud de sus trabajadores y clientes. Esto implicó la provisión de elementos de protección e higiene, readecuar sus locales, instalaciones y espacios y activar otras cuestiones operativas. Fue necesario dar capacitaciones al personal, preparar videos institucionales y mantener una comunicación abierta con sus empleados. Un entrevistado comentó: "Los colaboradores no son parte del problema si no de las soluciones, ellos se involucran, aportan ideas y se sienten contenidos si sus jefes los escuchan..."

Un caso interesante es el referido a una empresa que presta servicios de limpieza. Por razones obvias, en la actualidad sus servicios son muy demandados. Además, durante este tiempo, sus clientes les pidieron un nuevo producto "la sanitización" de sus locales, oficinas y hasta de casas particulares. Aprovechando esta demanda, capacitaron a su personal para incorporar y brindar este servicio en forma eficiente. Este es un claro ejemplo de cómo una empresa debe pensarse a partir de las necesidades insatisfechas del cliente y no simplemente de lo que sabe hacer.

Una situación que merece destacarse es el de aquellas empresas -esenciales o noque decidieron reforzar o implementar el trabajo a distancia, teletrabajo o home office. Esta nueva modalidad significó un cambio significativo para las organizaciones, si bien algunas lo aplicaban parcialmente, hubo necesidad de adaptar los sistemas y la tecnología a una forma de trabajo diferente.

La gente, tanto líderes como seguidores, tuvo rol fundamental. En algunos casos, refirieron que fueron los directivos quienes se sintieron más temerosos de enfrentar el cambio. No obstante, en general, la mayoría de los directivos evidenciaron una actitud proclive al cambio procurando trasmitir confianza y comunicación fluida con su personal. Los trabajadores mostraron respuestas diversas, muchos se adaptaron con facilidad a esta nueva modalidad, aunque para algunos significó un mayor estrés, sobre todo aquellos con hijos pequeños, falta de espacio en sus hogares y/o escaso manejo de la tecnología. Incluso, hubo algunos que rogaron volver a la oficina por falta de comodidad en sus casas. Unos pocos mostraron falta de colaboración y se adaptaron simplemente porque no les quedó otra opción.

En todos los casos consultados, los entrevistados revelaron que no hubo diferencias de aceptación entre los trabajadores de distintas generaciones. Un joven empresario comentó: "Este cambio en la modalidad de trabajo fue increíble, sin la pandemia todavía estaríamos discutiendo. Subimos quince peldaños en una semana”. 
En este grupo de casos, considerados esenciales que permanecieron abiertos desde el inicio de la pandemia, no afectados por medidas de aislamiento extremo, los directivos acompañaron el proceso de cambio con actitud proactiva, de apoyo, procurando seguir y crecer.

Este panorama situacional fue registrado hasta mediados de julio de 2020, pero el virus aún no se ha podido controlar. Como dijo el célebre compositor Enrique Santos Discépolo "el mundo sigue andando" y las organizaciones también, pero para subsistir hay que tener los ojos bien abiertos. Algunos se preguntan ¿este cambio vino para quedarse?, se presume que "no", nada perdura. Las modalidades y formas de trabajo deberán adaptarse o transformarse porque nada será igual.

\section{A MODO DE REFLEXIONES}

Entender y asumir los cambios no es tarea sencilla. Desde el marco conceptual, los autores del nuevo milenio invitan a las organizaciones a ser resilientes y a reinventarse, sin embargo, esta práctica no es cómoda ni habitual, especialmente en las pequeñas empresas que se perciben amenazadas por el entorno y limitadas en sus capacidades de gestión.

El muestreo de casos considerado para este trabajo, si bien no fue aleatorio fue heterogéneo, lo cual posibilitó tener una perspectiva amplia y diversa de los modos de trabajar y las formas de adaptación de sus miembros. Todos los casos estudiados experimentaron algún tipo de cambio, con grados de impactos diferentes. En general las organizaciones se adaptaron a la tecnología. Las que sucumbieron fue por impedimentos normativos para ejecutar sus prácticas laborales. Es decir, que el mayor escollo derivó la imposibilidad para trabajar no por falta de adaptabilidad de las personas a la tecnología.

Asumiendo que la supervivencia de las organizaciones depende de varios factores, no solo de la capacidad de gestión en situaciones de crisis, no es factible aseverar la carencia de capacidades de conducción de sus directivos y/o líderes. Tal vez las causales atribuidas al fracaso o extinción de las organizaciones se deban tanto a situaciones externas como a la carencia de políticas públicas que respalden a las pequeñas y medianas empresas, pero por el momento son solo hipótesis que requieren corroborarse.

Se comprobó que en el mundo de las empresas y el trabajo, los cambios se aceleraron por y con la pandemia, dejando numerosos interrogantes en torno al impacto Pospandemia. ¿Podrán las organizaciones en crisis recuperarse? Las que queden en pie ¿cómo se verán afectadas en sus estrategias, culturas y estructuras? 


\section{DISCUSIÓN}

Es oportuno comentar que el equipo de investigación se cuestiona en cuanto a la modalidad de exploración a través de videoconferencia. Si bien, la forma virtual se cree ventajosa como primera aproximación del estudio de casos, más considerando que fue la única alternativa para acceder a la información, se presume que resulta insuficiente.

Para profundizar en el estudio de casos y en la producción de conocimientos es preciso adicionar otros instrumentos de relevamiento. La observación personal en el campo y las entrevistas presenciales enriquecen las investigaciones aportando mayor y más detallada información para la comprensión de significados y el análisis de datos que, a los ojos del entrevistador resultan irremplazables por la pantalla.

$\mathrm{Si}$ bien este argumento no fue objeto de estudio, es trascendental porque hace al proceso de investigación y, como investigadoras atentas al rigor metodológico, no se puede dejar de cuestionar ni pasar por alto.

\section{REFERENCIAS BIBLIOGRÁFICAS}

Altschul, C. y R. Carbonell (2003). Transformando. Prácticas de cambio en empresas argentina. Buenos Aires: Eudeba.

BBC, entrevista por Lissardy, G. (05 de mayo, 2020) "Coronavirus: los 2 grandes escenarios mundiales que plantean algunos expertos para después de la pandemia", News Mundo, Nueva York, disponible en: https://www.bbc.com/mundo/noticias-internacional-52526090 [última visita: 01/07/2020].

Boggino, F. (2015). Trabajar o teletrabajar: ser productivos y felices, esa es la cuestión. Ciudad Autónoma de Buenos Aires: Metrópolis. También disponible en: https://recursos2puntocero.com/recursos/pdf/G TT QUEES.pdf

CEPAL (02 julio, 2020). "Sectores y Empresas frente al Covid-19". Informe Especial № 4 Covid-19, 02 de julio 2020. Disponible en: https://repositorio.cepal.org/bitstream/handle/11362/45734/4/S2000438 es.pdf [última visita: 13/08/2020].

Chiavenato, I. (2006). Introducción a la teoría general de la administración. [7ma. ed.]. México, D.F.: Mc Graw Hill.

Hamel, G. y C.K. Prahalad (1995). Compitiendo por el futuro. Buenos Aires: Ariel.

Hatum A. y N. J. Isola (22 agosto 2020). “La reinvención de los recursos humanos. El desafío de gestionar personas en la pandemia” Disponible en: 
https://www.utdt.edu/ver nota prensa.php?id nota prensa=18709\&id item m enu=6+\%20la\%20naci\%C3\%B3n [última visita: 25/08/2020].

Kobila, M.T. [et al.] (2019) "El cambio en las organizaciones. El caso de una Mutual Aseguradora" Ponencia presentada en las Vigésimascuartas Jornadas de Investigación de la Facultad de Ciencias Económicas y Estadística de la U.N.R, Rosario, noviembre de 2019. Disponible en: https://www.fcecon.unr.edu.ar/webnueva/actas-jornadas-anuales-de-investigacion

Laloux, F. (2017). Reinventar las organizaciones. [5ta.ed.]. Barcelona, España: Arpa.

Melamed, A. (2017). El futuro del trabajo y el trabajo del futuro. Ciudad Autónoma de Buenos Aries: Planeta.

Melamed, A. (22 julio, 2020) “Liderando el mañana” video de Conscious Business Center-Fred Kofman- https://www.youtube.com/watch?v=0NavCRgwzFk [última visita: 30/07/2020].

Molinari, P. (2017). Desencajados: herramientas para el nuevo mundo del trabajo. Ciudad Autónoma de Buenos Aires: Temas Grupo Editorial.

Molinari, P. (01 octubre, 2019) “Liderando en épocas de incertidumbre” Disponible en: https://www.glocalthinking.com/liderando-en-epocas-de-incertidumbre [última visita: 13/08/2020].

ORGANIZACIÓN INTERNACIONAL DEL TRABAJO (OIT) (2020). "Cumbre Mundial La Covid 19 y el mundo del trabajo", julio 2020, disponible en:

https://www.ilo.org/global/topics/coronavirus/events/WCMS 747501/lang-es/index.htm\#: :text=La\%200IT\%20organiz\%C3\%B3\%20del\%201,trabajo\%20 despu\%C3\%A9s\%20de\%20la\%20pandemia [última visita: 05/08/2020]

Poe, Richard (2009). Ola 4: el Network marketing en el siglo XXI. Buenos Aires: Prima Editorial.

Robbins, S. P. y M. Coulter (2018) Administración. [13 ed.]. D.F., México: Pearson. 